\title{
GENERALIZED SET-VALUED STRONGLY NONLINEAR VARIATIONAL INEQUALITIES IN BANACH SPACES
}

\author{
Y. J. Cho, Y. P. FANG, N. J. HuANG, AND K. H. Kim
}

\begin{abstract}
In this paper, we introduce and study a new class of generalized strongly nonlinear variational inequalities with setvalued mappings. By using the KKM technique, we prove the existence and uniqueness of solution for this class of generalized setvalued strongly nonlinear variational inequalities in reflexive $\mathrm{Ba}-$ nach spaces. Our results include the main results of Verma [16], [17] as special cases.
\end{abstract}

\section{Introduction and preliminaries}

Variational inequality theory has become a rich source of inspiration in pure and applied mathematics. Variational inequalities not only have stimulated the new results dealing with nonlinear partial differential equations, but also have been used in a large variety of problems arising in mechanics, physics, optimization and control, nonlinear programming, economics, finance, regional, structural, transportation, elasticity, and engineering sciences, etc. In recent years, variational inequalities have been generalized and applied in various directions. For more details, we refer to [1]-[3], [4]-[20] and the references therein.

In 1998, Verma [17] introduced and studied a class of monotone nonlinear variational inequalities, and proved the existence and uniqueness of solution for this kind of variational inequalities in reflexive Banach

Received April 17, 2002. Revised October 12, 2002.

2000 Mathematics Subject Classification: 47H06, 49J30, 49J40.

Key words and phrases: strongly nonlinear variational inequality, set-valued mapping, generalized monotone type mapping, generalized Lipschitzian type mapping, the KKM mapping.

The first author was supported financially from the Korea Science and Engineering Foundation (KOSEF-2000-1-10100-003-3). 
spaces. Very recently, Huang, Fang and Cho [12] extended Verma's results to more generalized nonlinear mixed quasi-variational inequality with single-valued mappings in reflexive Banach spaces.

In this paper, we introduce and study a new class of generalized strongly nonlinear variational inequalities with set-valued mappings and discuss the solvability of this class of generalized set-valued strongly nonlinear variational inequalities in reflexive Banach spaces. Our results improve and extend the corresponding results of [16] and [17].

Throughout this paper, let $X$ be a real reflexive Banach space, $X^{*}$ be its dual space, and $K$ be a nonempty convex closed subset of $X$. Denote $\langle\omega, x\rangle=\omega(x)$ for all $\omega \in X^{*}$ and $x \in X$. Let $S, T: K \rightarrow 2^{X^{*}}$ be two set-valued mappings, $M: X^{*} \times X^{*} \rightarrow X^{*}$ be a single-valued mapping and $f: K \rightarrow R \cup\{+\infty\}$ be a proper convex functional. We consider the following problem:

For any $\omega \in X^{*}$, find $u \in K$ such that

$$
\sup _{x \in S u, w \in T u}\langle M(x, w)-\omega, v-u\rangle+f(v)-f(u) \geq 0
$$

for all $v \in K$.

Some special cases of the problem (1.1) as follows:

(I) If $M(x, y)=x-y$, then the problem (1.1) is equivalent to the following problem:

For any $\omega \in X^{*}$, find $u \in K$ such that

$$
\sup _{x \in S u, w \in T u}\langle x-w-\omega, v-u\rangle+f(v)-f(u) \geq 0
$$

for all $v \in K$.

(II) If $S, T: K \rightarrow X^{*}$ is two single-valued mappings, then the problem (1.1) is equivalent to the following problem:

For any $\omega \in X^{*}$, find $u \in K$ such that

$$
\langle M(S u, T u)-\omega, v-u\rangle+f(v)-f(u) \geq 0
$$

for all $v \in K$.

REMARK 1.1. For a suitable choice of $M, S, T$ and $f$, the problem (1.1) includes many kinds of known variational inequalities as special cases (see [16], [17] and the references therein).

In the sequel, we give some definitions. 
Definition 1.1. A mapping $S: K \rightarrow 2^{X^{*}}$ is said to be $\varphi$-p monotone with respect to the first argument of the mapping $M: X^{*} \times X^{*} \rightarrow X^{*}$ if there exist a mapping $\varphi:[0,+\infty) \rightarrow[0,+\infty)$ and a constant $p \geq 1$ such that

$$
\langle M(x, \cdot)-M(y, \cdot), u-v\rangle \geq \varphi(\|u-v\|)\|u-v\|^{p}
$$

for all $u, v \in K, x \in S u$ and $y \in S v$.

Definition 1.2. A mapping $S: K \rightarrow 2^{X^{*}}$ is said to be $\varphi$-p monotone if there exist a mapping $\varphi:[0,+\infty) \rightarrow[0,+\infty)$ and a constant $p \geq 1$ such that

$$
\langle x-y, u-v\rangle \geq \varphi(\|u-v\|)\|u-v\|^{p}
$$

for all $u, v \in K, x \in S u$, and $y \in S v$.

Definition 1.3. A single-valued mapping $S: K \rightarrow X^{*}$ is said to be $\varphi-p$ monotone with respect to the first argument of the mapping $M: X^{*} \times X^{*} \rightarrow X^{*}$ if there exist $\varphi:[0,+\infty) \rightarrow[0,+\infty)$ and a constant $p \geq 1$ such that

$$
\langle M(S u, \cdot)-M(S v, \cdot), u-v\rangle \geq \varphi(\|u-v\|)\|u-v\|^{p}
$$

for all $u, v \in K$.

Remark 1.2. For a suitable choice of $S, \varphi, M$ and $p$, Definition 1.1 includes many known definitions of monotone type mappings as special cases (see [16], [17]).

Definition 1.4. A mapping $T: K \rightarrow 2^{X^{*}}$ is said to be relaxed $\psi-p$ monotone with respect to the second argument of the mapping $M: X^{*} \times X^{*} \rightarrow X^{*}$ if, there exist a mapping $\psi:[0,+\infty) \rightarrow[0,+\infty)$ and a constant $p \geq 1$ such that

$$
\langle M(\cdot, w)-M(\cdot, z), u-v\rangle \geq-\psi(\|u-v\|)\|u-v\|^{p}
$$

for all $u, v \in K, w \in T u$ and $z \in T v$.

Definition 1.5. A mapping $T: K \rightarrow 2^{X^{*}}$ is said to be $\psi$-p Lipschitzian if there exist a mapping $\psi:[0,+\infty) \rightarrow[0,+\infty)$ and a constant $p \geq 1$ such that

$$
\langle w-z, u-v\rangle \leq \psi(\|u-v\|)\|u-v\|^{p}
$$

for all $u, v \in K, w \in T u$ and $z \in T v$. 
Definition 1.6. A single-valued mapping $T: K \rightarrow X^{*}$ is said to be relaxed $\psi$-p monotone with respect to the second argument of the mapping $M: X^{*} \times X^{*} \rightarrow X^{*}$ if there exist a mapping $\psi:[0,+\infty) \rightarrow$ $[0,+\infty)$ and a constant $p \geq 1$ such that

$$
\langle M(\cdot, w)-M(\cdot, z), u-v\rangle \geq-\psi(\|u-v\|)\|u-v\|^{p}
$$

for all $u, v \in K, w \in T u$ and $z \in T v$.

REMARK 1.3. For a suitable choice of $T, M, \psi$ and $p$, Definition 1.4 includes many known definitions of Lipschitzian type and relaxed monotone type mappings as special cases (see [16], [17]).

Definition 1.7. Let $E, F$ be two topological spaces. A mapping $G: E \rightarrow 2^{F}$ is said to be lower semicontinuous if, for any net $\left\{x_{\alpha}\right\} \subset$ $E, x_{\alpha} \rightarrow x$ and a point $y \in G(x)$, there exist a subnet $\left\{x_{\beta}\right\} \subset\left\{x_{\alpha}\right\}$ and $y_{\alpha} \in G\left(x_{\alpha}\right)$ such that $y_{\alpha} \rightarrow y$.

Definition 1.8. A mapping $S: K \rightarrow X^{*}$ is said to be hemicontinuous if, for all $x, y, z \in K$, the mapping $t \mapsto\langle S(x+t y), z\rangle$ is continuous on [0,1]. A mapping $T: K \rightarrow 2^{X^{*}}$ is said to be lower hemicontinuous if, for all $x, y, z \in K$, the set-valued mapping $t \mapsto\langle T(x+t y), z\rangle$ is lower semicontinuous on $[0,1]$.

\section{Main results}

Now, we give our main results in this paper.

THEOREM 2.1. Let $X$ be a real reflexive Banach space, $X^{*}$ be its dual space and $K$ be a nonempty convex closed subset of $X$. Let $S, T: K \rightarrow$ $2^{X^{*}}$ be two set-valued mappings satisfying (1.4) and (1.7), respectively, where mappings $\varphi, \psi:[0,+\infty) \rightarrow[0,+\infty)$ satisfy $\varphi(t) \geq \psi(t)$ for all $t \geq 0$, and there exists a constant $\delta>0$ such that $\varphi-\psi$ is bounded on $[0, \delta]$. If the mapping $v \mapsto M(S v, T v)$ is lower hemicontinuous and $f: K \rightarrow R \bigcup\{+\infty\}$ is a proper convex functional. Then, for any $\omega \in X^{*}$, $u \in K$ is a solution of the problem (1.1) if and only if $u \in K$ is a solution of the following problem:

Find $u \in K$ such that

$$
\begin{gathered}
\langle M(y, z)-\omega, v-u\rangle+f(v)-f(u) \\
\geq(\varphi(\|v-u\|)-\psi(\|v-u\|))\|v-u\|^{p}
\end{gathered}
$$

for all $v \in K, y \in S v$ and $z \in T v$. 
Proof. Suppose that the problem (1.1) holds. Since the mappings $S$ and $T$ satisfy (1.4) and (1.7), respectively, then, for all $u, v \in K, x \in$ $S u, y \in S v, w \in T u$ and $z \in T v$, we have

$$
\begin{aligned}
& \langle M(y, z)-\omega, v-u\rangle+f(v)-f(u) \\
= & \langle-\omega, v-u\rangle+\langle M(x, z)-M(y, z), u-v\rangle \\
& +\langle M(x, w)-M(x, z), u-v\rangle+f(v)-f(u)-\langle M(x, w), u-v\rangle \\
\geq & \langle M(x, w)-\omega, \eta(v, u)\rangle+f(v)-f(u) \\
& +(\varphi(\|v-u\|)-\psi(\|v-u\|))\|v-u\|^{p} .
\end{aligned}
$$

Since $u \in K$ is a solution of the problem (1.1), this implies that

$$
\langle M(x, w)-\omega, v-u\rangle+f(v)-f(u) \geq 0
$$

for all $v \in K, x \in S u$ and $w \in T u$. Thus, we have

$$
\begin{gathered}
\langle M(y, z)-\omega, v-u\rangle+f(v)-f(u) \\
\geq(\varphi(\|v-u\|)-\psi(\|v-u\|))\|v-u\|^{p}
\end{gathered}
$$

for all $v \in K, y \in S v$ and $z \in T v$, i.e., (2.1) is true.

Conversely, suppose that (2.1) holds. Without loss of generality, choose a point $v \in K$ such that $f(v)<+\infty$ and so $f(u)<+\infty$. Letting $v_{n}=\left(1-\frac{1}{n}\right) u+\frac{1}{n} v$ for $n=1,2,3 \cdots$, then $v_{n} \in K$ and $v_{n}-u=\frac{1}{n}(v-u)$ for all $n \geq 1$. For any $x \in S u$ and $w \in T u$, since the mapping $v \mapsto M(S v, T v)$ is lower hemicontinuous and $v_{n} \rightarrow u$ as $n \rightarrow \infty$, there exist subsequence $\left\{v_{n_{j}}\right\} \subset\left\{v_{n}\right\}, y_{n_{j}} \in S v_{n_{j}}$ and $z_{n_{j}} \in T v_{n_{j}}$ such that, for any $\tau \in X$,

$$
y_{n_{j}} \rightarrow x, \quad z_{n_{j}} \rightarrow w, \quad\left\langle M\left(y_{n_{j}}, z_{n_{j}}\right), \tau\right\rangle \rightarrow\langle M(x, w), \tau\rangle
$$

as $j \rightarrow \infty$. It follows from (2.1) that

$$
\begin{aligned}
& \left.\left\langle M\left(y_{n_{j}}, z_{n_{j}}\right)-\omega, v_{n_{j}}-u\right)\right\rangle+f\left(v_{n_{j}}\right)-f(u) \\
= & \frac{1}{n_{j}}\left\langle M\left(y_{n_{j}}, z_{n_{j}}\right)-\omega, v-u\right\rangle+f\left(v_{n_{j}}\right)-f(u) \\
\geq & \left(\varphi\left(\left\|v_{n_{j}}-u\right\|\right)-\psi\left(\left\|v_{n_{j}}-u\right\|\right)\right)\left\|v_{n_{j}}-u\right\|^{p} \\
= & \left(\frac{1}{n_{j}}\right)^{p}\left(\varphi\left(\frac{1}{n_{j}}\|v-u\|\right)-\psi\left(\frac{1}{n_{j}}\|v-u\|\right)\right)\|v-u\|^{p} .
\end{aligned}
$$


Since $f$ is convex and $v_{n_{j}}-u=\frac{1}{n_{j}}(v-u)$, from (2.3), we have

$$
\begin{aligned}
& \left\langle M\left(y_{n_{j}}, z_{n_{j}}\right)-\omega, v-u\right\rangle+f(v)-f(u) \\
\geq & \left(\frac{1}{n_{j}}\right)^{p-1}\left(\varphi\left(\frac{1}{n_{j}}\|v-u\|\right)-\psi\left(\frac{1}{n_{j}}\|v-u\|\right)\right)\|v-u\|^{p} .
\end{aligned}
$$

It follows from (2.2) and (2.4) that

$$
\langle M(x, w)-\omega, v-u\rangle+f(v)-f(u) \geq 0
$$

for all $v \in K, x \in S u$, and $w \in T u$. This completes the proof.

If $M(x, y)=x-y$ for all $x, y$ in $X^{*}$, then Theorem 2.1 reduces to the following:

Theorem 2.2. Let $X$ be a real reflexive Banach space, $X^{*}$ be its dual space and $K$ be a nonempty convex closed subset of $X$. Let $S, T$ : $K \rightarrow 2^{X^{*}}$ be two lower hemicontinuous set-valued mappings satisfying (1.5) and (1.8), respectively. Suppose that the mappings $\varphi, \psi, f$ are the same as in Theorem 2.1. Then $u \in K$ is a solution of the problem (1.2) if and only if $u \in K$ is a solution of the following problem:

For any $\omega \in X^{*}$, find $u \in K$ such that

$$
\begin{gathered}
\langle y-z-\omega, v-u\rangle+f(v)-f(u) \\
\geq(\varphi(\|v-u\|)-\psi(\|v-u\|))\|v-u\|^{p}
\end{gathered}
$$

for all $v \in K, y \in S v$ and $z \in T v$.

If $S, T: K \rightarrow X^{*}$ are two single-valued mappings, then Theorem 2.1 reduces to the following:

Theorem 2.3. Let $X$ be a real reflexive Banach space, $X^{*}$ be its dual space and $K$ be a nonempty convex closed subset of $X$. Let $S, T: K \rightarrow$ $X^{*}$ be two single-valued mappings satisfying (1.6) and (1.9), respectively. Suppose that the mappings $\varphi, \psi, f$ are the same as in Theorem 2.1 and the mapping $v \mapsto M(S v, T v)$ is hemicontinuous. Then $u \in K$ is a solution of the problem (1.3) if and only if $u \in K$ is a solution of the following problem:

Find $u \in K$ such that

$$
\begin{aligned}
& \langle M(S v, T v)-\omega, v-u\rangle+f(v)-f(u) \\
\geq & (\varphi(\|v-u\|)-\psi(\|v-u\|))\|v-u\|^{p}
\end{aligned}
$$

for all $v \in K$. 
Remark 2.1. Theorems 2.1 2.3 improve and extend Theorem 2.1 of [16] and Theorem 2.1 of [17].

In the sequel, we need the following definition and lemma for our further results.

Definition 2.1. A mapping $F: X \rightarrow 2^{X}$ is said to be a $K K M$ mapping if, for any $\left\{x_{1}, x_{2}, \ldots, x_{n}\right\} \subset X$,

$$
\operatorname{co}\left\{x_{1}, x_{2}, \ldots, x_{n}\right\} \subset \bigcup_{i=1}^{n} F\left(X_{i}\right) .
$$

Lemma 2.1. [4] Let $K$ be a nonempty subset of a topological vector space $E$ and $F: K \rightarrow 2^{E}$ be a $K K M$ mapping. If $F(x)$ is closed in $E$ for every $x$ in $K$ and there exists at least a point $x_{0} \in K$ such that $F\left(x_{0}\right)$ is compact, then

$$
\bigcap_{x \in K} F(x) \neq \emptyset
$$

Theorem 2.4. Let $X$ be a real reflexive Banach space, $X^{*}$ be its dual space and $K$ be a nonempty bounded closed convex subset of $X$. Let $M, S, T, \varphi$, and $\psi$ be the same as in Theorem 2.1. Suppose that $\varphi-\psi$ is continuous and $f: K \rightarrow R \bigcup\{+\infty\}$ is proper convex lower semicontinuous. Then the problem (1.1) has a solution. Furthermore, if $\varphi(t) \neq \psi(t)$ for all $t>0$, then the solution is unique.

Proof. We first prove the existence of solution of the problem (1.1). Define the set-valued mappings $F, G: K \rightarrow 2^{K}$ by

$$
\begin{gathered}
F(v)=\{u \in K:\langle M(x, w)-\omega, v-u\rangle+f(v)-f(u) \geq 0 \\
\text { for some } x \in S u, w \in T u\}
\end{gathered}
$$

and

$$
\begin{aligned}
G(v)=\{u \in K & :\langle M(y, z)-\omega, v-u\rangle+f(v)-f(u) \\
& \geq(\varphi(\|v-u\|)-\psi(\|v-u\|))\|v-u\|^{p} \\
& \text { for all } y \in S v, z \in T v\}
\end{aligned}
$$

for all $v \in K$, respectively. We show that $F$ is a KKM mapping. Assume that $F$ is not a KKM mapping. Then there exist $\left\{v_{1}, v_{2}, \ldots, v_{n}\right\} \subset K$ and $t_{i}>0, i=1,2, \ldots, n$, such that

$$
\sum_{i=1}^{n} t_{i}=1, \quad v=\sum_{i=1}^{n} t_{i} v_{i} \notin \bigcup_{i=1}^{n} F\left(v_{i}\right) .
$$


For any $y \in S v$ and $z \in T v$, by the definition of $F$, we have

$$
\left\langle M(y, z)-\omega, v_{i}-v\right\rangle+f\left(v_{i}\right)-f(v)<0
$$

for $i=1,2, \ldots, n$. It follows that

$$
\begin{aligned}
0 & =\langle M(y, z)-\omega, v-v\rangle=\left\langle M(y, z)-\omega, \sum_{i=1}^{n} t_{i} v_{i}-v\right\rangle \\
& =\sum_{i=1}^{n} t_{i}\left\langle M(y, z)-\omega, v_{i}-v\right\rangle\left\langle\sum_{i=1}^{n} t_{i}\left(f(v)-f\left(v_{i}\right)\right)\right. \\
& =f(v)-\sum_{i=1}^{n} t_{i} f\left(v_{i}\right) \leq f(v)-f(v)=0,
\end{aligned}
$$

which is a contradiction. This implies that $F$ is a KKM mapping. Now we prove that $F(v) \subset G(v)$ for all $v \in K$. Let $u \in F(v)$, then there exist $x \in S u, w \in T u$ such that

$$
\langle M(x, w)-\omega, v-u\rangle+f(v)-f(u) \geq 0 .
$$

Since the mappings $S$ and $T$ satisfy (1.4) and (1.7), respectively, we have

$$
\begin{aligned}
& \langle M(y, z)-\omega, v-u\rangle+f(v)-f(u) \\
= & \langle M(x, z)-M(y, z), u-v\rangle+\langle M(x, w)-M(x, z), u-v\rangle \\
& +\langle-\omega, v-u\rangle-\langle M(x, w), u-v\rangle+f(v)-f(u) \\
\geq & (\varphi(\|v-u\|)-\psi(\|v-u\|))\|v-u\|^{p} \\
& +\langle M(x, w)-\omega, v-u\rangle+f(v)-f(u) \\
\geq & (\varphi(\|v-u\|)-\psi(\|v-u\|))\|v-u\|^{p}
\end{aligned}
$$

for all $v \in K, y \in S v$ and $z \in T v$. This implies that $u \in G(v)$, and so $G$ is also a KKM mapping. From the assumptions, we know that $G(v)$ is weakly closed for all $v$ in $K$. Since $K$ is bounded closed convex, we know that $K$ is weakly compact and so $G(v)$ is weakly compact in $K$ for all $v \in K$. It follows from Lemma 2.1 that

$$
\bigcap_{v \in K} G(v) \neq \emptyset
$$

Hence, there exists a point $u_{0} \in K$ such that

$$
\left\langle M(y, z)-\omega, v-u_{0}\right\rangle \geq\left(\varphi\left(\left\|v-u_{0}\right\|\right)-\psi\left(\left\|v-u_{0}\right\|\right)\right)\left\|v-u_{0}\right\|^{p}
$$


for all $v \in K, y \in S v$ and $z \in T v$. By Theorem 2.1, we know that

$$
\sup _{x_{0} \in S u_{0}, w_{0} \in T u_{0}}\left\langle M\left(x_{0}, w_{0}\right)-\omega,\left(v-u_{0}\right\rangle+f(v)-f\left(u_{0}\right) \geq 0\right.
$$

for all $v \in K$.

To show the uniqueness of the solution, letting $u_{1}, u_{2} \in K$ be two solutions of the problem (1.1). For any $x_{1} \in S u_{1}, w_{1} \in T u_{1}, x_{2} \in S u_{2}$ and $w_{2} \in T u_{2}$, we have

$$
\left\langle M\left(x_{1}, w_{1}\right)-\omega, v-u_{1}\right\rangle+f(v)-f\left(u_{1}\right) \geq 0
$$

and

$$
\left\langle M\left(x_{2}, w_{2}\right)-\omega, v-u_{2}\right\rangle+f(v)-f\left(u_{2}\right) \geq 0
$$

for all $v \in K$. Setting $v=u_{2}$ in (2.7) and $v=u_{1}$ in (2.8) and adding them, we have

$$
\left\langle M\left(x_{1}, w_{1}\right)-M\left(x_{2}, w_{2}\right), u_{2}-u_{1}\right\rangle \geq 0 .
$$

By (1.4) and (1.7), we obtain

$$
\begin{aligned}
& \left\langle M\left(x_{1}, w_{1}\right)-M\left(x_{2}, w_{2}\right), u_{2}-u_{1}\right\rangle \\
= & \left\langle M\left(x_{1}, w_{1}\right)-M\left(x_{2}, w_{1}\right), u_{2}-u_{1}\right\rangle \\
& +\left\langle M\left(x_{2}, w_{1}\right)-M\left(x_{2}, w_{2}\right), u_{2}-u_{1}\right\rangle \\
\leq & -\left(\varphi\left(\left\|u_{1}-u_{2}\right\|\right)-\psi\left(\left\|u_{1}-u_{2}\right\|\right)\right)\left\|u_{1}-u_{2}\right\|^{p} .
\end{aligned}
$$

Since $\varphi(t) \geq \psi(t)$ for all $t \geq 0$ and $\varphi(t) \neq \psi(t)$ for all $t>0$, it follows from (2.9) and (2.10) that

$$
\left\|u_{1}-u_{2}\right\|^{p}=0 .
$$

This implies that $u_{1}=u_{2}$. This completes the proof.

From Theorem 2.4, we have the following theorems:

Theorem 2.5. Let $X$ be a real reflexive Banach space, $X^{*}$ be its dual space and $K$ be a nonempty bounded closed convex subset of $X$. Let $S, T, \varphi, \psi$ and $f$ be the same as in Theorem 2.2. Suppose that $\varphi-\psi$ is continuous and $f: K \rightarrow R \bigcup\{+\infty\}$ is proper convex lower semicontinuous. Then the problem (1.2) has a solution. Furthermore, if $\varphi(t) \neq \psi(t)$ for all $t>0$, then the solution is unique. 
Theorem 2.6. Let $X$ be a real reflexive Banach space, $X^{*}$ be its dual space and $K$ be a nonempty bounded closed convex subset of $X$. Let the mappings $M, S, T, \varphi, \psi$ and $f$ be the same as in Theorem 2.3. The problem (1.3) has a solution. Furthermore, if $\varphi(t) \neq \psi(t)$ for all $t>0$, then the solution is unique.

REMARK 2.2. Theorems $2.4 \sim 2.6$ improve and extend Theorem 2.4 of [17].

\section{References}

[1] R. P. Agarwal, Y. J. Cho and N. J. Huang, Sensitivity analysis for strongly nonlinear quasi-variational inclusions, Appl. Math. Lett. 13 (2000), no. 6, 1924.

[2] Y. J. Cho, N. J. Huang and S. M. Kang, Random generalized set-valued strongly nonlinear implicit quasi-variational inequalities, J. Inequal. Appl. 5 (2000), no. $5,515-531$.

[3] S. S. Chang, Y. J. Cho, B. S. Lee and I. H. Jung, Generalized set-valued variational inclusions in Banach spaces, J. Math. Anal. Appl. 246 (2000), 409-422.

[4] K. Fan, Some properties of convex sets related to fixed point theorem, Math. Annal. 266 (1984), 519-537.

[5] F. Giannessi and A. Maugeri, Variational Inequalities and Network Equilibrium Problems, Plenum, New York, 1995.

[6] P. T. Harker and J. S. Pang, Finite-dimensional variational inequality and nonlinear complementarity problems: A survey of theory, algorithms and applications, Math. Programming 48 (1990), 161-220.

[7] N. J. Huang, On the generalized implicit quasi-variational inequalities, J. Math. Anal. Appl. 216 (1997), 197-210.

[8] __ A new completely general class of variational inclusions with noncompact valued mappings, Computers Math. Appl. 35 (1998), no. 10, 9-14.

[9] - Generalized nonlinear implicit quasi-variational inclusion and an application to implicit variational inequalities, Z. Angew. Math. Mech. 79 (1999), $569-575$.

[10] N. J. Huang, M. R. Bai, Y. J. Cho and S. M. Kang, Generalized nonlinear mixed quasi-variational inequalities, Computers Math. Appl. 40 (2000), no. 2-3, 205215.

[11] N. J. Huang and C. X. Deng, Auxiliary principle and iterative algorithms for generalized set-valued strongly nonlinear mixed variational-like inequalities, J. Math. Anal. Appl. 256 (2001), 345-359.

[12] N. J. Huang, Y. P. Fang and Y. J. Cho, A new class of generalized nonlinear mixed quasi-variational inequalities in Banach spaces, Math. Inequal. Appl. (to appear).

[13] N. J. Huang, Y. P. Liu, Y. Y. Tang and M. R. Bai, On the generalized set-valued strongly nonlinear implicit variational inequalities, Computers Math. Appl. 37 (1999), no. 10, 29-36. 
[14] P. D. Panagiotopoulos, Inequality Problems in Mechanics and Applications, Birkhäuser, Boston, 1985.

[15] M. V. Solodov and P. Tseng, Modified projection-type methods for monotone variational inequalities, SIAM J. Control. Optim. 34 (1996), no. 5, 1814-1836.

[16] R. U. Verma, Nonlinear variational inequalities on convex subsets of Banach spaces, Appl. Math. Lett. 10 (1997), no. 4, 25-27.

[17] , On monotone nonlinear variational inequality problems, Comment. Math. Univ. Carolinae 39 (1998), no. 1, 91-98.

[18] - Generalized pseudo-contractions and nonlinear variational inequalities, Publ. Math. Debrecen 53 (1998), no. 1-2, 23-28.

[19] G. X. Z. Yuan, KKM Theory and Applications, Marcel Dekker, New York, 1999.

[20] E. Zeidler, Nonlinear Functional Analysis and Its Applications IV, SpringerVerlag, New York, 1988.

\section{Y. J. Cho}

Department of Mathematics

Gyeongsang National University

Chinju 660-701, Korea

E-mail: yjcho@nongae.gsnu.ac.kr

Y. P. Fang and N. J. Huang

Department of Mathematics

Sichuan University

Chengdu, Sichuan 610064, P. R. China

E-mail: nic2601@scu.edu.cn

K. H. Kim

Department of Mathematics

Gyeongsang National University

Chinju 660-701, Korea 